\title{
The Diagnostic Role of Normalized and Mean Apparent Diffusion Coefficient in Differentiation between Pancreatic Lesions
}

Mai M. K. Barakat*, Laila Ahmad Abdurrahman, Doaa Anany, Nivan Hany Khater

Department of Radiology, Faculty of Medicine, Ain Shams University, Cairo, Egypt

*Correspondence author: Mai Mokhtar Kamal Barakat, Mobile: (+20)01006294780, E-mail: mai_mokhtar85@yahoo.com

\begin{abstract}
Background: Diffusion-weighted magnetic resonance imaging (DWI) with quantification of apparent diffusion coefficient (ADC) values is well established in the diagnosis of a variety of abdominal abnormalities. Regarding pancreatic disease, several investigators have shown that DWI with ADC measurement helps detect and characterize focal pancreatic lesions, as well as assess the severity of other pancreatic conditions.

Objective: The study aimed to evaluate the diagnostic role of both normalized and mean apparent diffusion coefficient in discrimination between pancreatic lesions.

Patients and Methods: Thirty-one participants presented with pancreatic lesions using clinical examination and ultrasound. Dynamic contrast MRI abdomen with diffusion-weighted MR imaging (DWI) on a 1.5-Tesla MRI machine was done. Mean ADC and normalized ADC (as the ratio of ADC of the lesion to the adjacent normal pancreas) were measured and compared.

Results: Our study reported a mean ADC cut value of $\leq 1.47$ while the cut-off value for normalized ADC was $\leq 0.96$. Normalized ADC revealed a higher sensitivity $92.31 \%$, specificity $88.89 \%$, PPV 85.71 , NPV $94.12 \%$, accuracy $90.32 \%$, positive likelihood ratio 8.31 and negative likelihood ratio 0.09 as compared to mean ADC, which revealed sensitivity $84.62 \%$, specificity $77.78 \%$, PPV $73.33 \%$, NPV $87.5 \%$, accuracy $80.65 \%$, positive likelihood ratio 3.81 and negative likelihood ratio 0.2 respectively.

Conclusion: Measuring the mean and normalized ADC value in pancreatic focal lesions can significantly differentiate between benign and malignant pancreatic lesions. However, normalized ADC has a higher sensitivity, specificity, PPV, and NPV than mean ADC value and could be used to differentiate between pancreatic lesions with higher accuracy than mean ADC.
\end{abstract}

Keywords: Diffusion-weighted MR imaging, Apparent diffusion coefficient, MRI, Normalized ADC, Pancreatic lesions.

\section{INTRODUCTION}

MRI is a known imaging modality for the evaluation and characterization of pancreatic masses. It is used for adequate analysis of the changes in the morphology of pancreatic parenchyma. However, there are many contraindications to the infusion of contrast agents due to its many adverse effects. Subsequently a need for another modality such as DWI to give the same diagnostic information ${ }^{(1)}$.

Diffusion-weighted imaging is an important sequence that could be used complementary to the main MRI protocol in pancreatic imaging. It could help in pancreatic focal lesions characterization and for diffuse parenchymal diseases evaluation. It is not time consuming and with no need for contrast media administration. Also, it could give us information about the cellularity of tissues and cell membranes status ${ }^{(2)}$. The water molecules' random motion is measured from DWI acquisitions in biologic tissues. The high cellularity and integrity of cell membranes suppress the motion-free water in both extracellular and intracellular compartments. So, tumors with high cellularity act as barriers to this free diffusion in both extra and intracellular compartments ${ }^{(3)}$.

$\mathrm{ADC}$ is a quantitative value calculated from DWI acquisitions. Malignant focal lesions have increased cellularity and show restricted diffusion on high $b$ value DWIs, with accompanying low ADC in comparison to normal tissue. However, the ADC values in the normal pancreas could vary between different scanners, protocols, and different parameters. Moreover, there is interobserver high variability due to the difficulty in the region of interest (ROIs) placement in the small glands. Therefore, normalized ADC has been introduced for more accurate differentiation of lesions ${ }^{(2)}$. Normalized ADC value might decrease the effects of many factors on calculating ADC, for example, the different strengths of field, $\mathrm{b}$ values, and variability of patients ${ }^{(3)}$.

The aim of the study was to evaluate the diagnostic value of measuring the normalized and mean $\mathrm{ADC}$ as a quantitative tool in differentiation between pancreatic masses.

\section{PATIENTS AND METHODS}

Our prospective study was performed in our institution from April 2018 till January 2020. Thirtyone patients (16 females and 15 males) were included in this study. Their ages ranged from 29 to 76 years with a mean age of $51.7 \pm 11.88$ years. They were diagnosed with pancreatic mass $1^{\text {st }}$ clinically followed by ultrasound. All were subjected to proper history taking. Patients were told to fast for 4 hours before the MRI 
examination in order to optimize visualization of the pancreaticobiliary tree. The MRI study was done for all patients, after written consent was taken from all of them.

Exclusion criteria: Patients with contraindication to contrast media (CM) e.g. elevated serum creatinine $(\geq 2$ $\mathrm{mg} / \mathrm{dl})$, decreased glomerular filtration rate (GFR) $(\leq 30$ $\mathrm{ml} / \mathrm{min}$ ), and participants with contraindication to MRI such as a non-MRI compatible pacemaker.

\section{The technique of MRI examination:}

MRI examination was done for all patients using 1.5 T Philips Achieva or GE electric 1.5 T MRI by using a body phased-array coil. Initial imaging included axial T2-weighted fast spin-echo (T2-WI) with fat suppression with a repetition time (TR) $2100 \mathrm{~ms}$, echo time (TE) $89.2 \mathrm{~ms}$, the field of view (FOV) $34.0 \mathrm{~cm}$, slice thickness, $4.0 \mathrm{~mm}$ and matrix, 256 $\times 224)$. As regards contrast study, T1-weighted dynamic contrastenhanced imaging (T1-WI) with fat suppression (fast spoiled gradient echo recalled acquisition in the steady state TR $210 \mathrm{~ms}$, TE $1.7 \mathrm{~ms}$, FOV $34.0 \mathrm{~cm}$, flip angle $80^{\circ}$ and bandwidth $31.25 \mathrm{kHz}$. DWI was acquired from the pancreas at different 20 slices using a finger pulse triggered diffusion-weighted single-shot spin-echo echo-planar imaging (EPI) technique (TR, the time between R-peaks (R-R) $\times 7 \mathrm{~ms}$, TE $63.5 \mathrm{~ms}, \mathrm{~b}=0,500$ and $1000 \mathrm{~s} / \mathrm{mm}^{2}$, RR interval, 7, trigger window $20 \%$, trigger delay minimum, inter-sequence delay minimum, cardiac phases single FOV $34.0 \mathrm{~cm}$, slice thickness 5 $\mathrm{mm}$, spacing $1 \mathrm{~mm}$, asset factor 2, NEX 8 and matrix, $128 \times 128)$.

All axial images were reconstructed to $256 \times 256$ matrix images after scanning. The lesion's mean ADC value was detected by drawing the region of interest over the detected lesion. The ROI was traced along the lesion boundaries by using an electronic cursor. It was placed manually to ensure that it was smaller than the actual lesion and so it was not including the adjacent normal tissue. The observer had placed region of interest (ROIs) to include as much as possible of the pancreatic lesion on the DWI obtained with $b=0$ $\mathrm{s} / \mathrm{mm}^{2}$. Other circular ROIs with a minimum size of 100 pixels were placed on the pancreatic normal segment with special care in a ROI placement to avoid visualized pancreatic vessels, ducts, and artifacts. Then the ROIs were copied from the b0 images to the ADC maps by using the "copy and paste" through the workstation. Then the ADC values were calculated automatically. Normalized ADC was calculated as the ratio of the lesion ADC to adjacent ADC of apparently normal pancreas.
Evaluation of the features of the lesions was recorded and included the size, margin, shape, signal intensity, and enhancement pattern in the dynamic contrast imaging as well as the site of the detected lesions. The provisional diagnosis was reported. Then, the diffusion images were reviewed with ADC values for final radiological characterization of the pancreatic lesions.

\section{Ethical consent:}

An approval of the study was obtained from Ain Shams University Academic and Ethical Committee. Reference Number of approval: FMASU 154. FWA 000017585. Every patient signed an informed written consent for acceptance of the study. This work has been carried out in accordance with The Code of Ethics of the World Medical Association (Declaration of Helsinki) for studies involving humans.

\section{Statistical analysis}

The data analysis was performed using IBM SPSS statistics V. 25.0 (IBM Corp, USA, 2017). Data were expressed as mean \pm standard deviation and median (IQR). Receiver operator curve (ROC) analysis was used to determine thresholds and the accuracy of the mean and normalized ADC in distinguishing the different groups. The diagnostic accuracy was evaluated as regards sensitivity, specificity, positive likelihood ratio, negative likelihood ratio, positive predictive value, negative predictive value, accuracy, and area under the curve. P-value $\leq 0.05$ was considered significant.

\section{RESULTS}

Thirty-one participants (16 females and 15 males) were included in this study. Their ages ranged from 29 to 76 years with a mean age of $51.7 \pm 11.88$ years.

Pathological analysis diagnosed that 18 lesions were benign (58\%) and 13 lesions (41.9\%) were malignant. The malignant lesions comprised 11 adenocarcinomas (AC) (Fig. 1, 2) and 2 mucinous cystadenocarcinomas. The benign lesions comprised of 7 pancreatic pseudocysts (PC), 3 mucinous cystadenomas (MCA) (Fig. 3), 3 necrotizing pancreatitis, one insulinoma (Fig. 4), one intraductal papillary mucinous neoplasm (IPMN), one splenule, one solid papillary tumor and one serous cystadenoma (SCA). 


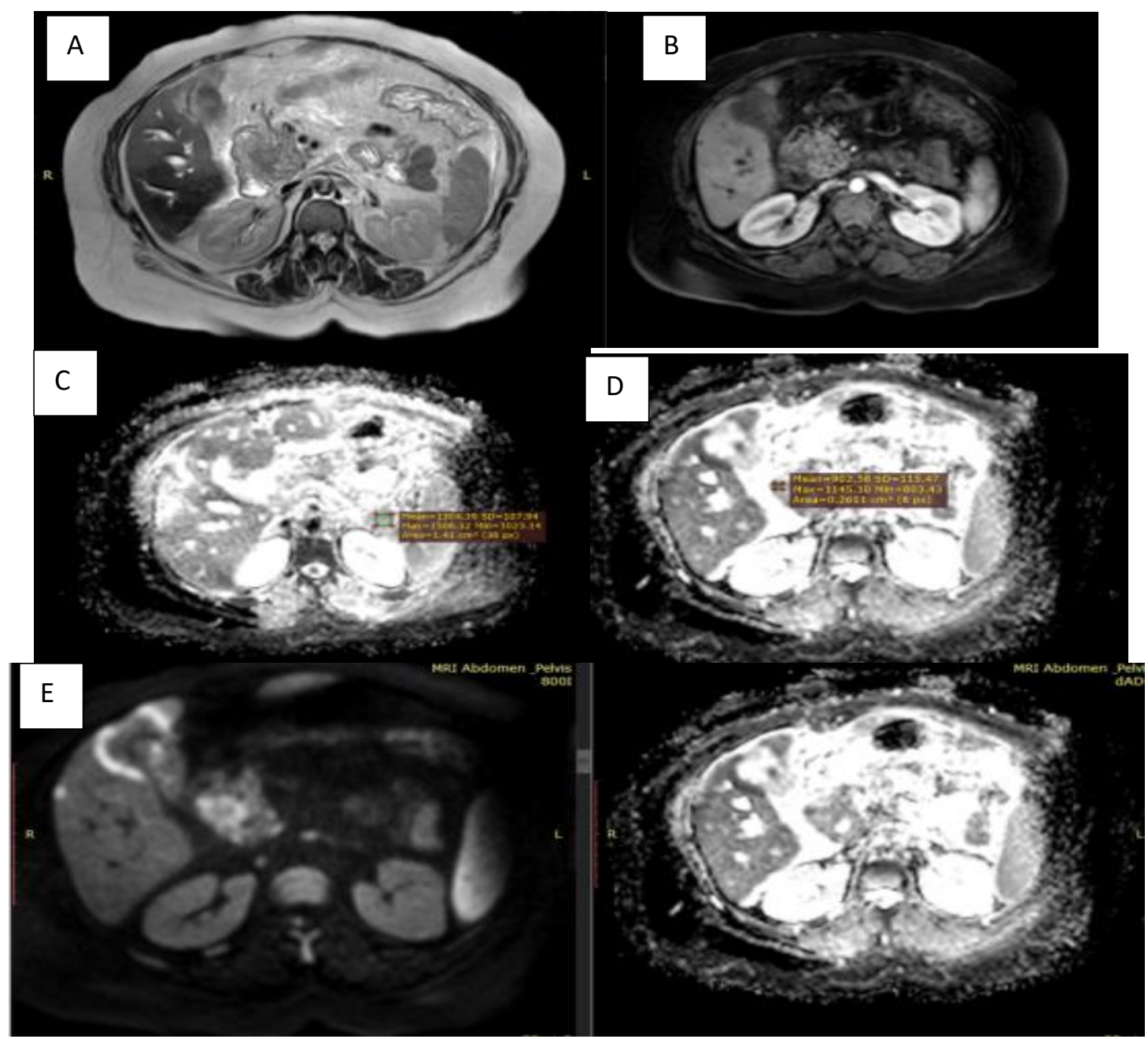

Figure (1): A 39 -year- old female presenting with progressive abdominal pain and anorexia. (A) T2WI, (B) T1 WI post contrast (C) ADC value of normal pancreas, (D) Mean ADC value of the lesion, (E) DWI and ADC. MRI findings: soft tissue mass lesion involving the head of the pancreas and non-separable from the duodenum with consequent IHBRD. It exhibited high SI in T2WI and heterogeneous delayed enhancement in the post-contrast study. On DWIs: The lesions appear bright. On the ADC map, the lesions turned dark denoting restricted diffusion. ADC value: Mean ADC $0.902 \times 10-3 \mathrm{~mm}^{2} / \mathrm{sec}$. Normalized ADC: $0.69 \times 10-3 \mathrm{~mm}^{2} / \mathrm{sec}$. Pathology of the lesion: Ductal adenocarcinoma.

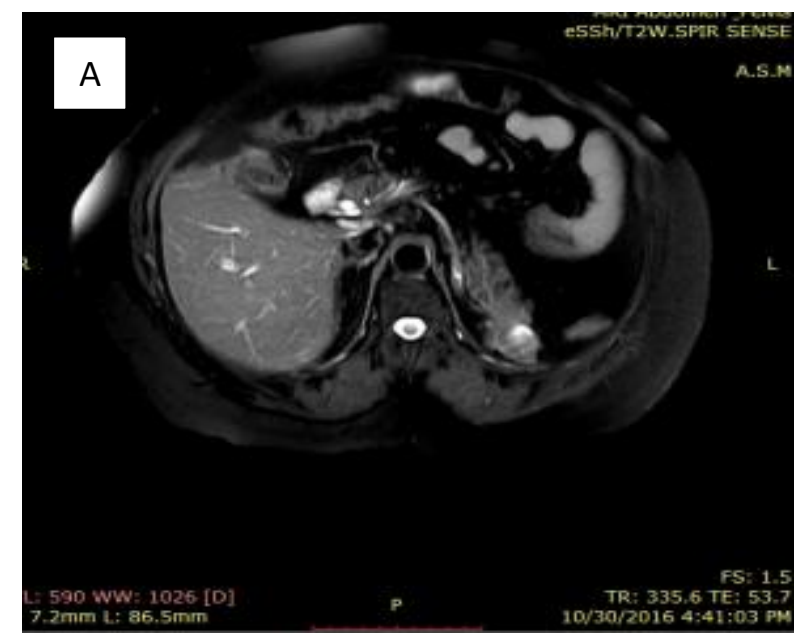




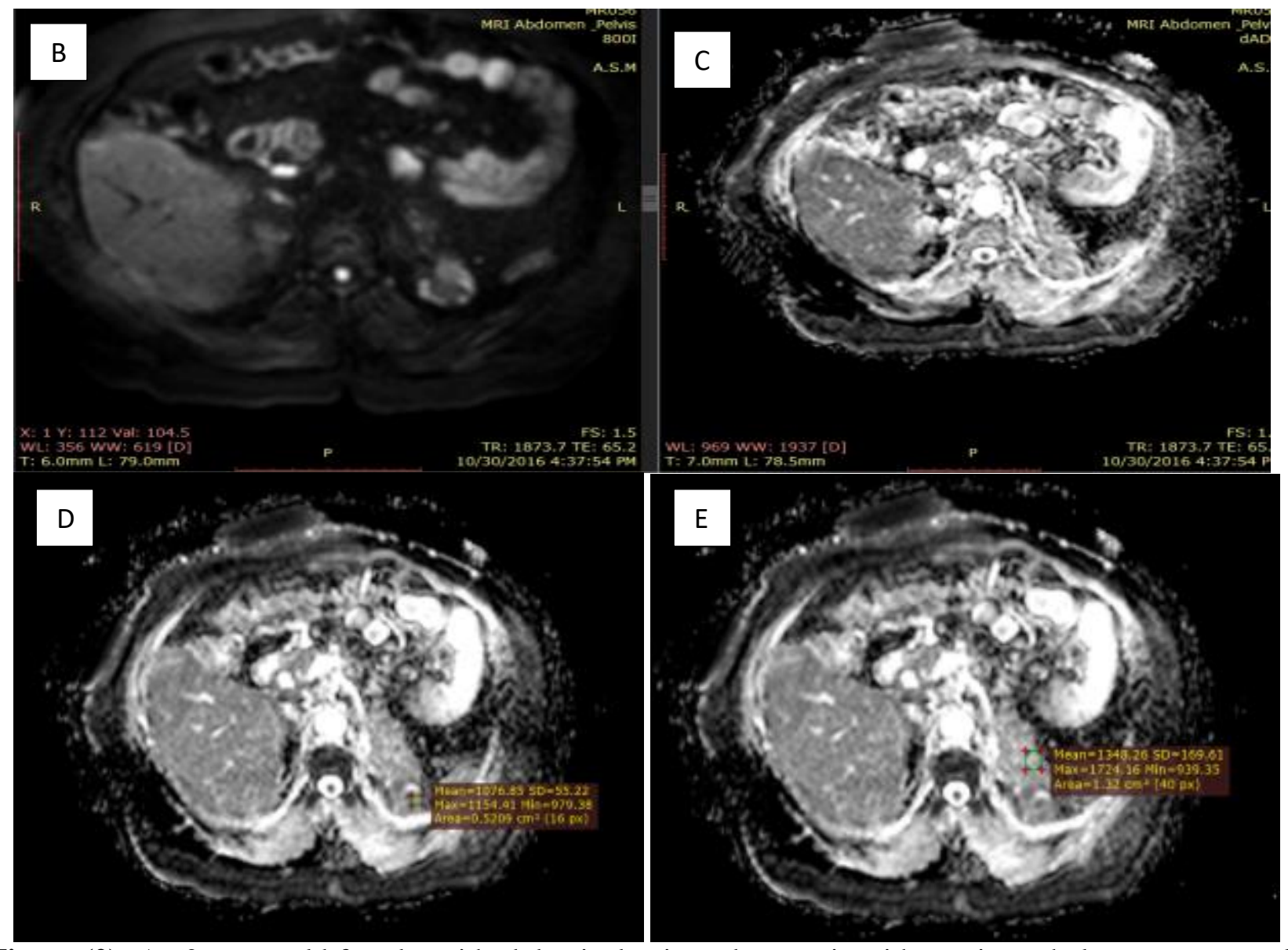

Figure (2): A 60- year- old female, with abdominal pain and anorexia with previous cholecystectomy, splenectomy, and left nephrectomy, (A) T2WI. (B) DWI and ADC. (C) Mean ADC (D) ADC of normal pancreas. MRI findings in figure (2) revealed a well-defined soft tissue lesion at the pancreatic tail with heterogeneous signal in T2WI denoting restricted diffusion and low ADC. ADC value: Mean ADC: 1.07 normalized ADC: 0.79. Pathology: pancreatic tail adenocarcinoma.

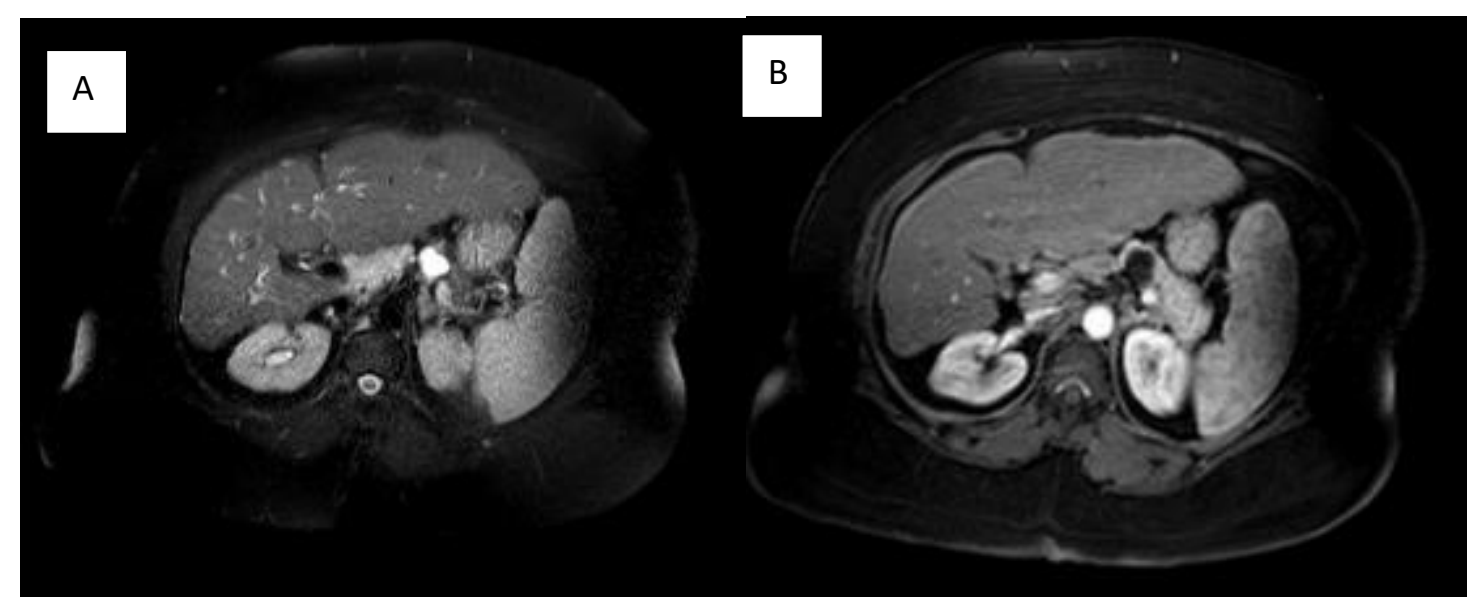




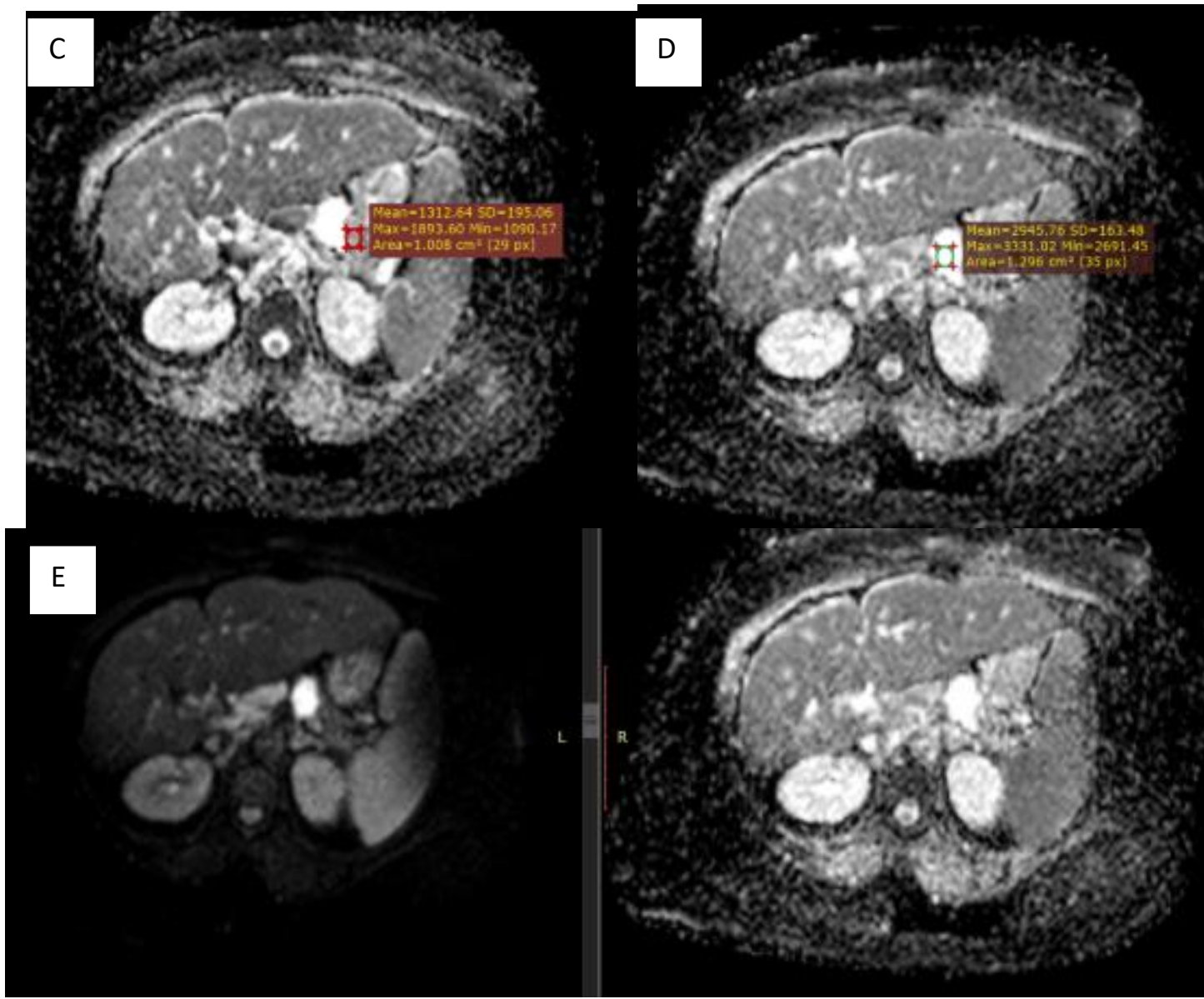

Figure (3): A 47 -year- old female patient complaining of vague abdominal pain. (A) T2WI, (B) T1 WI post-contrast (C) ADC value of normal pancreas, (D) Mean ADC value of the lesion, (E) DWI and ADC. MRI findings in figure (3) showed a well-defined pancreatic body cystic lesion that elicited a high signal in T2 with no enhancement in the postcontrast study. On DWIs: The lesions appear bright. On the ADC map, the lesion was still bright, indicating the T2 shine-through effect (no true diffusion restriction). ADC value: Mean ADC: 2.945 x 10-3 m²/sec, normalized ADC: $2.24 \times 10-3 \mathrm{~mm}^{2} / \mathrm{sec}$. Pathology of the lesion revealed: Mucinous Cystadenoma.

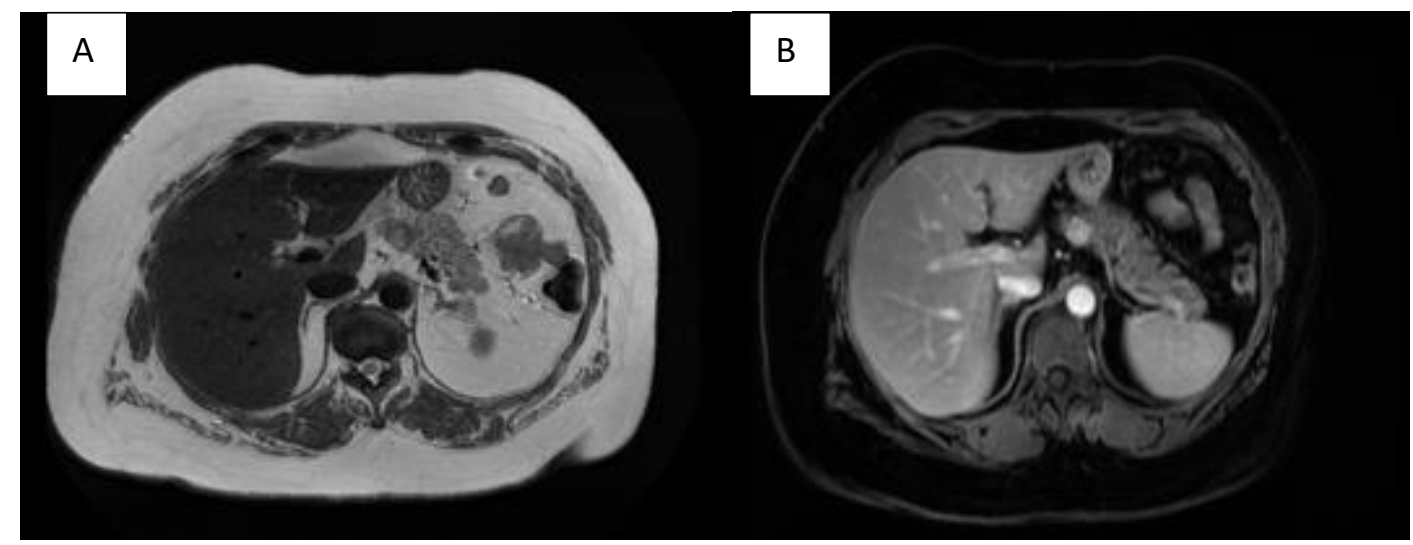




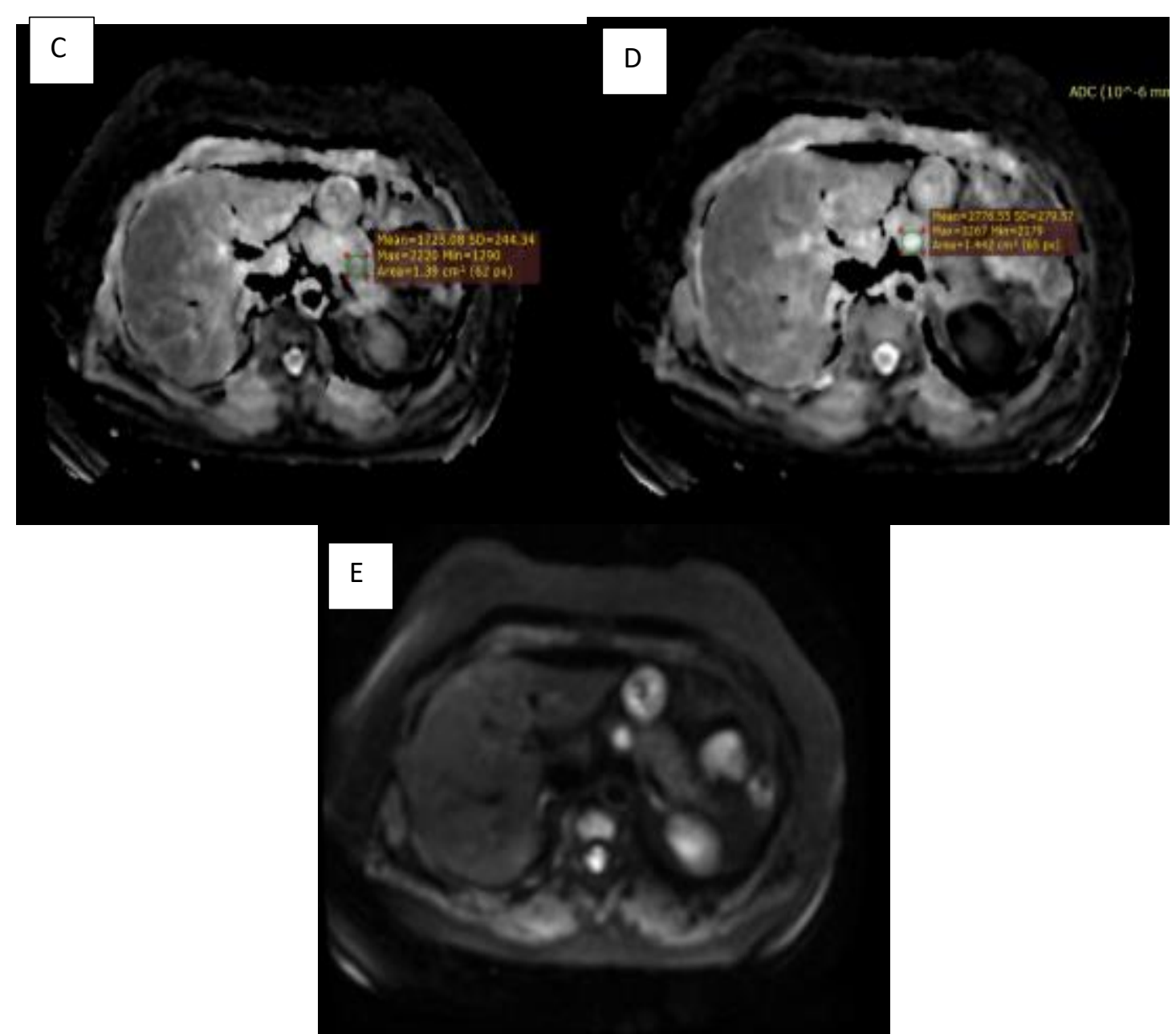

Figure (4): A 59- year- old female presented with hypoglycemia and abdominal pain. (A) T2WI, (B) T1 WI postcontrast (C) ADC value of normal pancreas, (D) Mean ADC value of the lesion, (E) DWI. MRI findings in figure (4) revealed a well-defined focal mass at the pancreatic neck opposing the ventral surface of the superior mesenteric artery without evidence of occlusion or invasion. The mass is posterior to the pancreatic duct with no evidence of duct abnormality. It displays a bright T2WI signal with progressive post-contrast enhancement. On DWIs: The lesion appeared bright. On the ADC map, the lesion was still bright signal, denoting the T2 shine-through effect. ADC value: Mean ADC: 2.776 x 10-3 mm²/sec. Normalized ADC: $1.6 \times 10-3 \mathrm{~mm}^{2} / \mathrm{sec}$. Pathology of the lesion revealed: NET, insulinoma.

Eight of the adenocarcinomas, the SPT and the insulinoma were solid on imaging, 2 mixed solid and cystic lesions while all the remaining lesions were purely cystic. The sizes of the pancreatic lesions ranged from $1.1-13 \mathrm{~cm}$ with a mean of $4.51 \pm 3.01 \mathrm{~cm}$. Both benign and malignant lesions could be differentiated using both mean and normalized $A D C$ values. Malignant tumors revealed significant lower normalized $A D C$ measurements $(P=0.005)$ and mean ADC measurements $(\mathrm{P}=0.008)$. Normalized ADC for benign lesions revealed a mean of 1.71), \pm 0.53 and a median of 1.89. On the other hand, mean $\mathrm{ADC}$ revealed a mean of $2.59 \pm 0.98$ and a median of 2.62. As regards malignant lesions, normalized ADC for benign lesions revealed a mean of $0.75 \pm 0.118$ and a median of 0.72 . On the other hand, mean ADC revealed a mean of $1.17 \pm 0.26$ and a median of 1.13 (Table 1 ). 
Table (1): Mean and normalized ADC values for benign and malignant groups.

\begin{tabular}{|c|c|c|c|}
\hline \multirow{3}{*}{$\begin{array}{l}\text { Mean } \\
\text { ADC }\end{array}$} & \multirow{2}{*}{\multicolumn{2}{|c|}{ Benign }} & \multirow{2}{*}{$\frac{\text { Statistic }}{2.5933}$} \\
\hline & & & \\
\hline & & Median & 2.6200 \\
\hline & & Std. Deviation & 0.98023 \\
\hline & & Minimum & 1.12 \\
\hline & & Maximum & 4.30 \\
\hline & & Range & 3.17 \\
\hline & & Interquartile Range & 1.82 \\
\hline & \multirow[t]{7}{*}{ Malignant } & Mean & 1.1723 \\
\hline & & Median & 1.1300 \\
\hline & & Std. Deviation & 0.26929 \\
\hline & & Minimum & 0.91 \\
\hline & & Maximum & 1.70 \\
\hline & & Range & 0.80 \\
\hline & & Interquartile Range & 0.36 \\
\hline \multirow{14}{*}{$\begin{array}{l}\text { Normalized } \\
\text { ADC }\end{array}$} & \multirow[t]{7}{*}{ Benign } & Mean & 1.7111 \\
\hline & & Median & 1.8900 \\
\hline & & Std. Deviation & 0.53799 \\
\hline & & Minimum & 0.78 \\
\hline & & Maximum & 2.52 \\
\hline & & Range & 1.75 \\
\hline & & Interquartile Range & 1.01 \\
\hline & \multirow[t]{7}{*}{ Malignant } & Mean & 0.7500 \\
\hline & & Median & 0.7200 \\
\hline & & Std. Deviation & 0.11853 \\
\hline & & Minimum & 0.62 \\
\hline & & Maximum & 1.03 \\
\hline & & Range & 0.42 \\
\hline & & Interquartile Range & 0.15 \\
\hline
\end{tabular}

Data were expressed as mean $\pm \mathrm{SD}$, median, interquartile range and range.

The normalized ADC sensitivity was $92.31 \%$, specificity was $88.89 \%$, PPV was 85.71 , NPV was $94.12 \%$, accuracy was $90.32 \%$, positive likelihood ratio was 8.31 and negative likelihood ratio was 0.09 . The mean ADC sensitivity was $84.62 \%$, specificity was $77.78 \%$, PPV was $73.33 \%$, NPV was $87.5 \%$, accuracy was $80.65 \%$, positive likelihood ratio was 3.81 and negative likelihood ratio was 0.2 (Table 2).

Table (2): The cut-off value, sensitivity, specificity, accuracy, negative likelihood ratio (NLR), positive likelihood ratio (PLR), positive predictive value (PPV) and negative predictive value (NPV) of the mean, and normalized ADC

\begin{tabular}{|c|c|c|c|c|c|c|c|c|}
\hline Parameter & $\begin{array}{c}\text { Optimu } \\
\text { m cut off } \\
\text { value }\end{array}$ & Sensitivity & Specificity & PLR & NLR & NPV & PPV & Accuracy \\
\hline Mean ADC & $\leq 1.47$ & $92.31 \%$ & $88.89 \%$, & 8.31 & 0.09 & $94.12 \%$ & $85.71 \%$ & $90.32 \%$ \\
\hline $\begin{array}{c}\text { Normalized } \\
\text { ADC }\end{array}$ & $\leq 0.96$ & $84.62 \%$ & $77.78 \%$ & 3.81 & 0.2 & $87.5 \%$ & $73.33 \%$ & $80.65 \%$ \\
\hline
\end{tabular}

We used a mean $\mathrm{ADC}$ threshold of $\leq 1.47$, subsequently, there were 14 true negative cases, 11 true positives, two cases were false negative (cystic adenocarcinoma and mucinous cystadenocarcinoma) and 4 cases were false positive (2 necrotizing pancreatitis, 1 spenule, and 1 solid papillary tumor ). We used a normalized ADC threshold of $\leq 0.96$. So, there were 16 true negative cases, 12 true positives, one case was false negative (mucinous cystadenocarcinoma), and 2 cases were false positive ( 1 spenule and 1 solid papillary tumor).

The area under the curve (AUC) for normalized ADC was higher than that of the mean ADC. The AUC for mean ADC was 0.938 (95\% CI, 0.857-1.000), and for normalized ADC was 0.966 (95\% CI, 0.912-1.000) respectively. However, there was no statistically significant difference between them (p-value 0.343) (Fig. 5). 


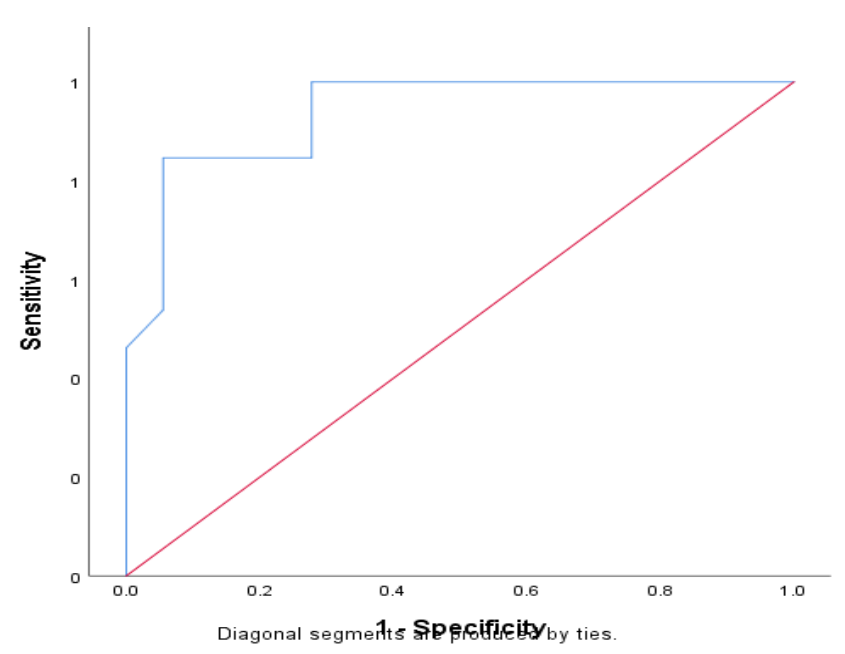

(A) Mean ADC

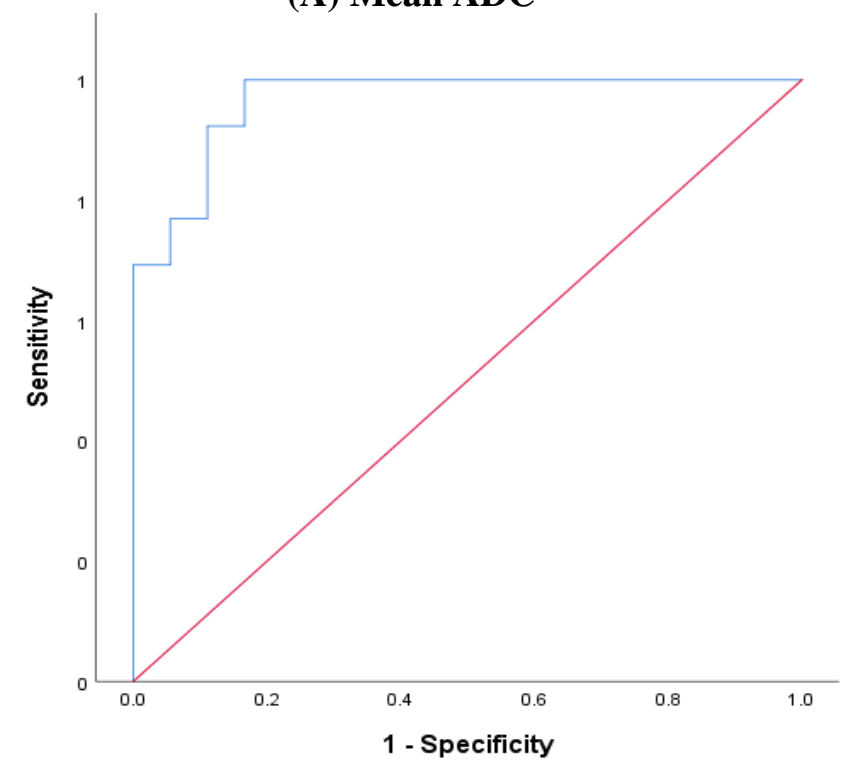

(B) Normalized ADC

Figure (5): (A) Mean ADC. (B) Normalized ADC. The receiver operating characteristic curve analyses. The specificity, sensitivity and AUC using mean (A) and normalized $\mathrm{ADC}(\mathrm{B})$ values for differentiation between benign and malignant pancreatic lesions.

\section{DISCUSSION}

ADC is a quantitative value, which could be calculated from DWI acquisitions. Most of Malignant focal lesions have increased cellularity and subsequently show restricted diffusion on high $b$ value DWIs, with accompanying low ADC in comparison with normal tissue. The ADC values in the normal pancreas could vary between different scanners, protocols, and different parameters. Moreover, there is interobserver high variability due to the difficulty in the ROIs placement in the small gland. Therefore, normalized ADC has been introduced for optimum differentiation of lesions ${ }^{(2)}$. A study done by Ding $\boldsymbol{e t} \boldsymbol{a l}$. (3) showed that there was no statistically significant difference $(\mathrm{P}>.05)$ in the measurement of the mean and normalized $\mathrm{ADC}$ at the different pancreatic anatomical sites. Subsequently, in our study we measured the mean and normalized ADC values of the pancreatic lesions and normal pancreatic tissue at any anatomical site avoiding the pancreatic ducts and vascular structures.
Normalized ADC was calculated as the ratio of pancreatic lesion ADC to adjacent pancreas ADC, which was apparently normal.

To our knowledge, few studies used both normalized and mean ADC values to differentiate between pancreatic lesions. In our study, the pancreatic lesions were categorized as benign or malignant, both groups could be discriminated using both mean and normalized $\mathrm{ADC}$ values. Benign and malignant tumors showed statistically significantly different mean ADC values $(\mathrm{P}=0.008)$ and normalized $\mathrm{ADC}$ values $(\mathrm{P}=$ 0.005). A preliminary study done by Barral et al. ${ }^{(4)}$ measured the normalized and conventional ADC in pancreatic lesions revealed similar statistically significant differences in conventional ADC values between benign and malignant tumors with $\mathrm{p}$-value $(\mathrm{P}$ $=0.004$ ). Additionally, normalized ADC values in the same study showed significant differences between malignant tumors and benign tumors with $\mathrm{p}$ - value $(\mathrm{P}<$ 0.0001).

We reported a mean ADC cut-off value measuring about $\leq 1.47$ with a sensitivity of $84.62 \%$, specificity of $77.78 \%$, PPV of $73.33 \%$, NPV of $87.5 \%$, accuracy of $80.65 \%$, positive likelihood ratio of 3.81 , and negative likelihood ratio of 0.2. Additionally, we reported the normalized ADC cut off value measuring $\leq$ 0.96 with a sensitivity of $92.31 \%$ specificity of $88.89 \%$, PPV of 85.71 , NPV of $94.12 \%$, accuracy of $90.32 \%$, positive likelihood ratio of 8.31 , and negative likelihood ratio of 0.09 . According to our results, the normalized ADC had a higher sensitivity, specificity, PPV, and NPV than compared to mean ADC. However, no significant difference was found between the area under the curve (AUC) for the mean and normalized ADC in discriminating between benign and malignant lesions with p-value ( $\mathrm{P}=0.343)$. Yet, the AUC for normalized $\mathrm{ADC}$ was higher than that of the mean ADC. In concordance to our study, a study done by Barral $\boldsymbol{e t}$ al. (4) gave the same results that normalized ADC values of pancreatic lesions had higher degrees of discrimination between pancreatic lesions than the conventional mean ADC.

A lower comparable cut-off result of Mean $\mathrm{ADC}$ values had been reported in different studies. Most of the studies were done on $1.5 \mathrm{~T}$ machines such as Muraoka et al. ${ }^{(5)}$ and Abdallah et al. ${ }^{(6)}$.

They measured the mean ADC values and there was a statistically significant difference in the mean ADC values between malignant and noncancerous normal tissue in these two studies. In both studies, the mean value was lower than our study measuring about (1.27) and was significantly lower than for noncancerous tissue. Abdallah et al. ${ }^{\left({ }^{6}\right)}$ revealed higher sensitivity (95.5\%) and PPV (91.3\%), yet lesser specificity $75 \%$ and NPV $85.7 \%$ than our study. A lower sensitivity (72\%) and specificity (76.9\%) for mean ADC has been reported by Lee $\boldsymbol{e t}$ al. ${ }^{(7)}$ using a near cut-off value of about 1.33 . They used only two $b$ values for DWI (0 and $\left.500 \mathrm{sec} / \mathrm{mm}^{2}\right)$. 
In contrast to Elteeh et al. ${ }^{(8)}$ the cut-off value of mean ADC was lower than our study (about 1.37) with higher sensitivity, specificity, and negative predictive values of DWI 92\%, 97\%, 98\% as well as PPV $85 \%$ respectively.

Few studies were done on $3 \mathrm{~T}$ machine, Huang et al. ${ }^{(9)}$ revealed a lower threshold for ADC about 1.1 with higher sensitivity and specificity. They used only two $b$ values of 0 and $1000 \mathrm{sec} / \mathrm{mm}^{2}$. While a study done by Fukukura et al. ${ }^{\left({ }^{(10)}\right.}$ reported the mean ADC of the tumors was about 1.16. However, a study done by Park et al. ${ }^{(11)}$ revealed a higher sensitivity $(96 \%-98 \%)$ by two observers yet it was a qualitative study using only DWI without measuring ADC values. Variations in ADC values may be related to the differences in population, different imaging sequences, and different specific $b$ values slice thickness or the strength of the magnetic field.

To our knowledge, researchers reported that the higher b-values on DW imaging shows more signal hyperintensity in pancreatic adenocarcinomas in comparison with the surrounding tissue or other benign lesions ${ }^{\left({ }^{(10)}\right.}$. Our study was performed on 1.5 tesla MRI machine and we used several $b$-values $(b=0,500$ and 1000) $\mathrm{sec} / \mathrm{mm}^{2}$ in the acquisition of DWI. Many authors used the same $\mathrm{b}$ values on the same $1.5 \mathrm{~T}$ machines in their studies such as Suliman et al. ${ }^{(1)}$,

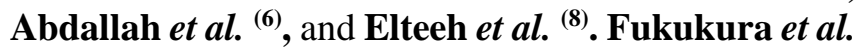
(10) used a b value of $1000 \mathrm{sec} / \mathrm{mm}^{2}$ on a $3 \mathrm{~T}$ machine while Ma et al. ${ }^{(12)}$ used 0 and $600 \mathrm{~b}$ values on a 3T machine. On the other hand, Fattahi et al. ${ }^{(13)}$ used the same b-values of Ma et al. ${ }^{(12)}$ but on 1.5 T MRI. In contrast to Kamisawa et al. ${ }^{(14)}$ where they performed their study at $1.5 \mathrm{~T}$ using $\mathrm{b}$ values of 0,500 , and 800 $\mathrm{sec} / \mathrm{mm}^{2}$ while Barral et al. ${ }^{(\mathbf{1 5})}$ used a $1.5 \mathrm{~T}$ machine using 0, 400 , and $800 \mathrm{~b}$ values. Ding et al. ${ }^{(3)}$ used the same $b$ values of Huang et al. ${ }^{(9)}$ On a 3T machine with only 0 and $1000 \mathrm{~b}$ values. While the study of Tonolini and Di Pietro ${ }^{(2)}$ used four (b values $0,50,600,800$ ) on a $1.5 \mathrm{~T}$ machine.

There were several limitations in our study due to the limited number of cases and the use of a $1.5 \mathrm{~T}$ MRI machine and not 3T. A $2^{\text {nd }}$ limitation was that we didn't use the IVIM model to investigate the different abilities of the perfusion fraction and the diffusion perfusion free parameter. Furthermore, we didn't study the ability of ADC value in the determination of the degree of malignant tumors and if it could give a decision about tumor respectability. We further recommend investigating the effect of the different $\mathrm{b}$ values on the modification of normalized ADC.

\section{CONCLUSION}

Measuring the mean and normalized ADC values in pancreatic focal lesions can significantly differentiate between benign and malignant pancreatic lesions. However, normalized ADC has a higher sensitivity, specificity, PPV, and NPV than mean ADC value and could be used to differentiate between pancreatic lesions with higher accuracy than mean ADC. It can be used complementary to conventional MRI before histopathological biopsy.

\section{Financial support and sponsorship: Nil. Conflict of interest: Nil.}

\section{REFERENCES}

1. Abdel Kader M, Abass H, Suliman M (2019): Apparent diffusion coefficient of pancreatic adenocarcinoma: is there any congruity with tumor resectability? Egypt J Radiol Nucl Med., 50 (1): 1-9.

2. Tonolini M, Di Pietro S (2019): Diffusion-weighted MRI: New paradigm for the diagnosis of interstitial oedematous pancreatitis. Gland Surg., 8 (2): 197-206.

3. Ding X, Xu H, Zhou J et al. (2019): Reproducibility of normalized apparent diffusion coefficient measurements on 3.0-T diffusion-weighted imaging of normal pancreas in a healthy population. Medicine (Baltimore), 98 (14): 1510415111.

4. Barral M, Sebbag-Sfez D, Hoeffel C et al. (2013): Characterization of focal pancreatic lesions using normalized apparent diffusion coefficient at 1.5-Tesla: preliminary experience. Diagn Interv Imaging, 94 (6): 619-627.

5. Muraoka N, Uematsu H, Kimura H et al. (2008): Apparent diffusion coefficient in pancreatic cancer: Characterization and histopathological correlations. J Magn Reson Imaging, 27 (6): 1302-1308

6. Abdallah E, Ali M, Awadallah M et al. (2016): Role of diffusion weighted imaging in characterization of pancreatic pathology. Egypt J Radiol Nucl Med., 47 (3): 723-727.

7. Lee S, Byun J, Park B et al. (2008): Quantitative analysis of diffusion-weighted magnetic resonance imaging of the pancreas: usefulness in characterizing solid pancreatic masses. J Magn Reson Imaging, 28: 928-936.

8. Warda M, Hasan D, Elteeh O (2015): Differentiation of Pancreatic lesions using Diffusion-Weighted MRI. Egypt J Radiol Nucl Med., 46 (3): 563-568.

9. Huang W, Sheng J, Chen S et al. (2011): Differentiation between pancreatic carcinoma and mass-forming chronic pancreatitis: Usefulness of high $\mathrm{b}$ value diffusion-weighted imaging. J Dig Dis., 12 (5): 401-408.

10. Fukukura Y, Takumi K, Kamimura K et al. (2012): Pancreatic adenocarcinoma: variability of diffusionweighted MR imaging findings. Radiology, 263 (3): 732-40.

11. Park M, Kim Y, Choi S et al. (2014): Preoperative detection of small pancreatic adenocarcinoma: Value of adding diffusion-weighted imaging to conventional MR imaging for improving confidence level. Radiology, 273 (2): 433-443.

12. Ma C, Guo X, Liu L et al. (2017): Effect of region of interest size on ADC measurements in pancreatic adenocarcinoma. Cancer Imaging, 17 (1): 1-7.

13. Fattahi R, Balci N, Perman W et al. (2009): Pancreatic diffusion-weighted imaging (DWI): comparison between mass-forming focal pancreatitis (FP), pancreatic cancer (PC), and normal pancreas. J Magn Reson Imaging, 29: 350-356.

14. Kamisawa T, Takuma K, Anjiki H et al. (2010): Differentiation of autoimmune pancreatitis from pancreatic cancer by diffusion-weighted MRI. Am J Gastroenterol., 8: 1870-1875.

15. Barral M, Taouli B, Guiu B et al. (2015): Diffusionweighted MR imaging of the pancreas: Current status and recommendations. Radiology, 274 (1): 45-63. 